\title{
Ground state energy of trimmed discrete Schrödinger operators and localization for trimmed Anderson models
}

\author{
Alexander Elgart ${ }^{1}$ and Abel Klein ${ }^{2}$
}

\begin{abstract}
We consider discrete Schrödinger operators of the form $H=-\Delta+V$ on $\ell^{2}\left(\mathbb{Z}^{d}\right)$, where $\Delta$ is the discrete Laplacian and $V$ is a bounded potential. Given $\Gamma \subset \mathbb{Z}^{d}$, the $\Gamma$-trimming of $H$ is the restriction of $H$ to $\ell^{2}\left(\mathbb{Z}^{d} \backslash \Gamma\right)$, denoted by $H_{\Gamma}$. We investigate the dependence of the ground state energy $E_{\Gamma}(H)=\inf \sigma\left(H_{\Gamma}\right)$ on $\Gamma$. We show that for relatively dense proper subsets $\Gamma$ of $\mathbb{Z}^{d}$ we always have $E_{\Gamma}(H)>E_{\emptyset}(H)$. We use this lifting of the ground state energy to establish Wegner estimates and localization at the bottom of the spectrum for $\Gamma$-trimmed Anderson models, i.e., Anderson models with the random potential supported by the set $\Gamma$.
\end{abstract}

Mathematics Subject Classification (2010). Primary 82B44; Secondary 47B80, 60H25.

Keywords. Anderson models, trimmed Anderson models, discrete Schrödinger operators, random Schrödinger operators, ground state energy, Cheeger's inequality, localization, Wegner estimates.

\section{Introduction}

We consider discrete Schrödinger operators of the form $H=-\Delta+V$ on $\ell^{2}\left(\mathbb{Z}^{d}\right)$, where $\Delta$ is the discrete Laplacian, defined by

$$
(-\Delta \varphi)(x)=\sum_{\substack{y \in \mathbb{Z}^{d},\|x-y\|=1}}(\varphi(x)-\varphi(y))=2 d \varphi(x)-\sum_{\substack{y \in \mathbb{Z}^{d},\|x-y\|=1}} \varphi(y),
$$

and $V$ is a bounded potential. Given $\Gamma \subsetneq \mathbb{Z}^{d}$, the $\Gamma$-trimming of $H$ is the restriction $H_{\Gamma}$ of $\chi_{\Gamma^{\mathrm{c}}} H \chi_{\Gamma^{\mathrm{c}}}$ to $\ell^{2}\left(\Gamma^{\mathrm{c}}\right)$, where $\chi_{A}$ denotes the characteristic function of the set $A$ and $A^{\mathrm{c}}=\mathbb{Z}^{d} \backslash A$ for $A \subset \mathbb{Z}^{d}$. We focus our attention on $E_{\Gamma}(H)=\inf \sigma\left(H_{\Gamma}\right)$, the ground state energy (or bottom of the spectrum) of the trimmed discrete Schrödinger operator $H_{\Gamma}$. (Note that with this notation $H=H_{\emptyset}$ and $E_{\emptyset}(H)=\inf \sigma(H)$.) Since

\footnotetext{
${ }^{1}$ Supported in part by the NSF under grant DMS-1210982.

${ }^{2}$ Supported in part by the NSF under grant DMS-1001509.
} 
$E_{\Gamma}(H)$ is a nondecreasing function of the set $\Gamma$, trimming lifts the bottom of the spectrum, that is, $E_{\Gamma}(H) \geq E_{\emptyset}(H)$.

We show that for relatively dense proper subsets $\Gamma$ of $\mathbb{Z}^{d}$ we always have strict lifting of the bottom of the spectrum, i.e., $E_{\Gamma}(H)>E_{\emptyset}(H)$. We use this lifting of the ground state energy to establish Wegner estimates and localization at the bottom of the spectrum for $\Gamma$-trimmed Anderson models, i.e., Anderson models with the random potential supported by the set $\Gamma$.

1.1. The ground state energy of trimmed discrete Schrödinger operators. Our motivation comes from continuous Schrödinger operators $H=-\Delta+V$ on $\mathrm{L}^{2}\left(\mathbb{R}^{d}\right)$, where $\Delta$ is the Laplacian operator and $V$ is a bounded potential. Let us consider first the case $H=-\Delta$ and $\Gamma^{\mathrm{c}}$ an open subset of $\mathbb{R}^{d}$, and let $\Delta_{\Gamma}$ be the Laplacian on $\Gamma^{\mathrm{c}}$ with Dirichlet boundary condition. When $\overline{\Gamma^{\mathrm{c}}}$ is compact, the ground state energy $E_{\Gamma}(-\Delta)$ of $-\Delta_{\Gamma}$ is the first eigenvalue $\lambda_{\Gamma}$ of $-\Delta_{\Gamma}$. The problem of obtaining a lower bound for the first eigenvalue of the Dirichlet Laplacian on a compact Riemannian manifold has been intensively studied in Geometric Analysis, and it is given by Cheeger's inequality [7]: $\lambda_{\Gamma} \geq \frac{\beta(\Gamma)^{2}}{4}$, where $\beta(\Gamma)$ is Cheeger's isoperimetric constant for the set $\Gamma^{\mathrm{c}}$. It is known that $\beta(\Gamma)>0$ if $\overline{\Gamma^{\mathrm{c}}}$ is compact, but for noncompact sets $\overline{\Gamma^{\mathrm{c}}}$ the Cheeger isoperimetric constant may be zero.

Cheeger's inequality has been extended to the discrete case; see [12] and [28]. In this context, $\beta(\Gamma)=\inf _{S \subset \Gamma^{c}: 1 \leq|S|<\infty} \frac{|\partial S|}{|S|}$, where $\partial S$ denotes the boundary of the set $S$ and $|S|$ its cardinality. (See Section 2.3 for notation and details. An equivalent definition for $\beta(\Gamma)$ is given in (2.18)). Clearly $\beta(\Gamma)>0$ if $\Gamma^{\mathrm{c}}$ is a finite set. But it is not difficult to see that $\beta(\Gamma)=0$ if we can find a sequence of boxes in $\mathbb{Z}^{d}, \Lambda_{K_{n}}\left(x_{n}\right)\left(\Lambda_{K}(x)\right.$ is the box of side $K \in \mathbb{N}$ centered at $\left.x \in \mathbb{Z}^{d}\right)$, such that $\lim _{n \rightarrow \infty} \frac{\left|\Gamma \cap \Lambda_{K n}\left(x_{n}\right)\right|}{\left|\Gamma^{\mathrm{c}} \cap \Lambda_{K_{n}}\left(x_{n}\right)\right|}=0$. This lead us to consider relatively dense subsets $\Gamma$ of $\mathbb{Z}^{d}$, for which we show $\beta(\Gamma)>0$.

The addition of a potential $V$ breaks down Cheeger's argument. Indeed, in general flat functions are no longer good approximants for the low-lying eigenvectors of $H=-\Delta+V$. For example, let $H_{\lambda}=-\Delta+\lambda V$, where $V$ is a periodic potential whose average over a fundamental cell is equal to zero. Then $E_{\emptyset}\left(H_{\lambda}\right)<0$ for all $\lambda>0$, see Theorem 1 in [22] (the result there is proven for the continuum, but it is easy to see that holds in the discrete case as well), but it can be shown that $\beta_{\lambda}(\varnothing)=0$ for $\lambda$ small, where $\beta_{\lambda}(\varnothing)$ is the Cheeger constant for $H_{\lambda}$. Another striking counterexample can be constructed by taking $H_{\lambda}=-\Delta+\lambda V$ with $V=-\chi_{\{0\}}$, a negative rank one perturbation to $-\Delta$, and $\lambda>0$. It is well known that in this case $E_{\emptyset}\left(H_{\lambda}\right)<0$ for all $\lambda>0$, while it is easy to see that $\beta_{\lambda}(\emptyset)=0$ for $\lambda \leq 2 d$.

For continuous Schrödinger operators the bound $E_{\Gamma}(H)>E_{\emptyset}(H)$ can be established in the presence of an arbitrary bounded potential using the unique continuation principle; see [24] and [32]. Unfortunately, discrete Schrödinger operators do not satisfy a unique continuation principle. It turns out, however, that the ground state of a discrete Schrödinger operator $H$ enjoys a similar property, which suffices to 
establish the desired result.

It is intuitively clear that the Schrödinger operator $H_{\Gamma}$ is, in a suitable sense, the limit of the Schrödinger operators $H_{\Gamma}(t)=H+t \chi_{\Gamma}$ on $\ell^{2}\left(\mathbb{Z}^{d}\right)$ as $t \rightarrow \infty$. This is the motivation behind Theorem 1.1, where we obtain a lower bound for $E_{\Gamma}(H)-E_{\emptyset}(H)$ as the limit of lower bounds for $E_{\Gamma}(H, t)-E_{\emptyset}(H)$, where $E_{\Gamma}(H, t)=E_{\emptyset}(H(t))$. Note that $E_{\Gamma}(H, t)$ is nondecreasing in $t$, so $E_{\Gamma}(H, \infty):=\lim _{t \rightarrow \infty} E_{\Gamma}(H, t)=$ $\sup _{t \geq 0} E_{\Gamma}(H, t)$, and it follows from the min-max principle that $E_{\Gamma}(H, t) \leq E_{\Gamma}(H)$ for all $t \geq 0$, so

$$
E_{\emptyset}(H) \leq E_{\Gamma}(H, t) \leq E_{\Gamma}(H, \infty) \leq E_{\Gamma}(H) .
$$

Before stating our results, we introduce some additional notation. A bounded potential $V$ is given by multiplication by a a function $V: \mathbb{Z}^{d} \rightarrow \mathbb{R}$ with $V_{\infty}=$ $\|V\|_{\infty}<\infty$. We set $V_{+}=\max \{V, 0\}$ and $V_{-}=-\min \{V, 0\}$; note that $V=$ $V_{+}-V_{-}, V_{ \pm} \geq 0$, and $V_{+} V_{-}=0$. We define the spread of the bounded potential $V$ by

$$
\operatorname{spr}(V)=\sup _{x \in \mathbb{Z}^{d}} V(x)-\inf _{x \in \mathbb{Z}^{d}} V(x) \in[0, \infty) .
$$

We also introduce the notation

$$
\begin{gathered}
Y_{d, V}=2 d+1+\operatorname{spr}(V), \\
\delta_{\Gamma}(H)=E_{\Gamma}(H)-E_{\emptyset}(H), \\
\delta_{\Gamma}(H, t)=E_{\Gamma}(H, t)-E_{\emptyset}(H) .
\end{gathered}
$$

Theorem 1.1. Let $H=-\Delta+V$ be a Schrödinger operator on $\ell^{2}\left(\mathbb{Z}^{d}\right)$, where $V$ is a bounded potential, and let $\Gamma \subsetneq \mathbb{Z}^{d}$. Then

$$
E_{\Gamma}(H, \infty)=E_{\Gamma}(H) .
$$

Moreover, we have

$$
2 d+\operatorname{spr}(V) \geq \delta_{\Gamma}(H, t) \geq \frac{t}{t+6 d+2 \operatorname{spr}(V)} \delta_{\Gamma}(H), \quad t \geq 0 .
$$

It follows, using (1.1), that $E_{\Gamma}(H)>E_{\emptyset}(H)$ if and only if $E_{\Gamma}(H, t)>E_{\emptyset}(H)$ for all $t>0$.

Theorem 1.1 is proven in Section 2.1. Note that once we have a lower bound for $\delta_{\Gamma}(H)$, as in Theorem 1.3, (1.2) (we may use the sharper (2.4)) provides lower bounds for $\delta_{\Gamma}(H, t)$ for all $t>0$. 
Given $x \in \mathbb{Z}^{d}$ and $L>0$, we set

$$
\Lambda_{L}(x)=\left\{y \in \mathbb{Z}^{d}:\|y-x\|_{\infty} \leq \frac{L}{2}\right\}
$$

and

$$
\Lambda_{L}^{(0)}(x)=\left\{y \in \mathbb{Z}^{d}:\|y-x\|_{\infty}<\frac{L}{2}\right\}
$$

note that $\Lambda_{L}(x)=\Lambda_{L}^{(0)}(x) \Longleftrightarrow L \notin 2 \mathbb{N}$.

Given $K \in \mathbb{N}$ we have

$$
\left|\Lambda_{K}(x)\right|=K_{*}^{d},
$$

where

$$
K_{*}=\left\{\begin{array}{l}
K \text { if } K \text { is odd } \\
K+1 \text { if } K \text { is even. }
\end{array}\right.
$$

Moreover $\Lambda_{K}(x)=\Lambda_{K}^{(0)}(x)$ if and only if $K$ is odd.

Definition 1.2. A set $\Gamma \subset \mathbb{Z}^{d}$ is $(K, Q)$-relatively dense, where $K, Q \in \mathbb{N}$, if

$$
\left|\Gamma \cap \Lambda_{K}^{(0)}(\zeta)\right| \geq Q, \quad \zeta \in K \mathbb{Z}^{d} .
$$

By a relatively dense subset $\Gamma \subset \mathbb{Z}^{d}$ we will always mean a $(K, Q)$-relatively dense set $\Gamma$ for some appropriate $K, Q \in \mathbb{N}$. Note that we must have $Q \leq\left|\Lambda_{K}^{(0)}\right| \leq$ $K^{d}$, and that $K \geq 2$ unless $\Gamma=\mathbb{Z}^{d}$.

Theorem 1.3. Let $\Gamma \subsetneq \mathbb{Z}^{d}$ be $(K, Q)$-relatively dense, and let $H=-\Delta+V$ on $\ell^{2}\left(\mathbb{Z}^{d}\right)$, where $V$ is a bounded potential. Then

$$
\delta_{\Gamma}(H, t) \geq \frac{Q}{(2 d K-1) Y_{d, V}^{2 d K-1}}\left(1-\left(\frac{Y_{d, V}}{Y_{d, V}+t}\right)^{2 d K-1}\right), \quad t \geq 0 .
$$

As a consequence, we get

$$
\delta_{\Gamma}(H) \geq \frac{Q}{(2 d K-1) Y_{d, V}^{2 d K-1}}>0 .
$$

In the special case $H=-\Delta$ we can improve the previous bound to

$$
\delta_{\Gamma}(-\Delta)=E_{\Gamma}(-\Delta) \geq \frac{1}{4 d K_{*}^{2 d}} .
$$

We prove (1.3) from a 'quantitative unique continuation principle for ground states' given in Lemma 2.2. The lower bound given in (1.4) holds for arbitrary bounded potential $V$; note that it depends on $V$ only through $\operatorname{spr}(V)$. 
The special case (1.5) follows from a Cheeger's inequality. We remark that $E_{\Gamma}(-\Delta)$ can also be estimated by an argument of Bourgain and Kenig Section 4 in [5] (see also [21], Remark 4.4). They treated continuum models, but Rojas-Molina, see Section 1.2.5 in [30] and Lemma 2.1 in [31], noted that the argument applies also to the discrete case. This argument yields the bound $E_{\Gamma}(-\Delta) \geq \frac{C}{K^{2 d+2}}$.

Theorem 1.3 has a continuum counterpart; in particular we can use Cheeger's inequality to obtain the continuum version of (1.5). We did not include it in this paper because the continuous version of the general estimate (1.3) is only marginally better than the estimate in [24], Lemma 4.2, and the continuous version of (1.5) is similarly only marginally better that what we get with the Bourgain-Kenig argument.

Theorem 1.3 follows from Theorems 2.1 and 2.3 in Section 2.2.

1.2. Wegner estimates and localization for trimmed Anderson models. If $\zeta \in$ $\mathbb{Z}^{d}$, we will use the notation $\chi_{\zeta}$ for $\chi_{\{\zeta\}}$ as a multiplication operator in $\ell^{2}\left(\mathbb{Z}^{d}\right)$, but we will write $\delta_{\zeta}$ instead when considering $\chi_{\{\xi\}}$ as a function in $\ell^{2}\left(\mathbb{Z}^{d}\right)$.

\subsubsection{Trimmed Anderson models.}

Definition 1.4. Let $\Gamma \subset \mathbb{Z}^{d}$ be $(K, Q)$-relatively dense. A $\Gamma$-trimmed Anderson model is a discrete random Schrödinger operator on on $\ell^{2}\left(\mathbb{Z}^{d}\right)$ of the form

$$
H_{\omega, \lambda}:=H_{0}+\lambda V_{\omega}
$$

where

(i) $H_{0}=-\Delta+V^{(0)}$, with $V^{(0)}$ a bounded (background) potential;

(ii) $V_{\omega}$ is the random potential given by

$$
V_{\omega}:=\sum_{\zeta \in \Gamma} \omega_{\zeta} \chi_{\zeta},
$$

where $\omega=\left\{\omega_{\zeta}\right\} \zeta \in \Gamma$ is a family of independent random variables whose probability distributions $\left\{\mu_{\zeta}\right\} \xi \in \Gamma$ are non-degenerate with

$$
\operatorname{supp} \mu_{\zeta} \subset[0, M], \quad \zeta \in \mathbb{Z}^{d} ;
$$

(iii) $\lambda>0$ is the disorder parameter.

If $\Gamma=\mathbb{Z}^{d}, V^{(0)}=0$, and $\mu_{\zeta}=\mu$ for all $\zeta \in \mathbb{Z}^{d}$, then $H_{\omega, \lambda}$ is the standard Anderson model. This model was introduced by Anderson [4] to study the effect of disorder on electronic states within the suitable energy range. The main phenomenon is localization, which manifests itself as spectral localization (the spectral measure of $H_{\omega, \lambda}$ is almost surely pure point with exponential decay of eigenfunctions) and as dynamical localization (non-spreading of wave packets). 
Trimmed Anderson models are the discrete analogues of the crooked Anderson Hamiltonians introduced in [24], Definition 1.2. (By a trimmed Anderson model we will always mean a $\Gamma$-trimmed Anderson model for some relatively dense subset $\Gamma \subset \mathbb{Z}^{d}$.)

The standard Anderson model with sufficiently regular single site probability distribution $\mu$ was intensively studied during the last two decades; see [1], [2], [3], [11], [13], [14], [15], [16], [27], [34], and [35] and the reviews [20], [23], and [33] for a more exhaustive list of references. (In this paper we consider only results valid in arbitrary dimension $d$; the $d=1$ case is special and we will not mention $d=1$ only results.) It exhibits localization in an interval at the bottom of the spectrum for fixed disorder and on the whole real line for large disorder. On the other hand, until very recently there had been no localization results for ergodic $\Gamma$ - trimmed Anderson models with $\Gamma \neq \mathbb{Z}^{d}$, say $\Gamma=K \mathbb{Z}^{d}$ with $K \geq 2$. The reason is the lack of a covering condition, i.e., that the support of the random potential is all of $\mathbb{Z}^{d}$ with probability one. Indeed, $\sum_{\zeta \in K \mathbb{Z}^{d}} \chi_{\zeta}=\chi_{\Gamma}$, and hence $\sum_{\zeta \in K \mathbb{Z}^{d}} \chi_{\zeta} \geq c>0$ if and only if $\Gamma=\mathbb{Z}^{d}$. The covering condition has played a crucial role in the proofs of Wegner estimates (which are bounds on the regularity of the integrated density of states, first proved by Wegner [36] for the standard Anderson model) and localization for the Anderson model.

This difficulty has been overcome for the continuous analogue of the Anderson model by the use of the unique continuation principle for continuous Schrödinger operators, and localization at the bottom of the spectrum has been proved for continuous Anderson Hamiltonians; see [5], [9], [10], and [21]. These results were further extended to a larger class of continuous random Schrödinger operators with alloytype random potentials, including non-ergodic random Schrödinger operators such as Delone-Anderson Hamiltonians; see [24], [30], and [32].

Recently, Rojas-Molina [30], Theorem 1.2.6, proved Wegner estimates and localization at the bottom of the spectrum for the special case of $2 \mathbb{Z}^{d}$-trimmed Anderson models with no background potential, i.e., $V^{(0)}=0$. She circumvented the lack of covering condition using an argument of Bourgain and Kenig [5], Section 4, as described in [21], Remark 4.4. Her approach can be extended for $\Gamma$-trimmed Anderson models with $\Gamma$ an arbitrary relatively dense subset of $\mathbb{Z}^{d}$, as long as there is no background potential [31], Section 2.1; the Bourgain-Kenig argument does not appear to be able to incorporate a background potential. Cao and Elgart [6] showed localization at small disorder below the bottom of the free spectrum for a class of three-dimensional Anderson-like models without background potential. The "non overlapping setup' in [6] includes $k \mathbb{Z}^{d}$-trimmed Anderson models where the random variables are supported by the interval $[-1,1]$, but it is more general in that it admits finite rank random perturbations with non overlapping supports and no definite sign. Theorem 1 in [6] establishes not only localization at small disorder for this class of Anderson models, but also, using the Feynman diagrammatic technique, gives an explicit expression (as a function of the disorder) for the interval of localization. 
Although there is no unique continuation principle for discrete Schrödinger operators, we prove Wegner estimates and localization at the bottom of the spectrum for $\Gamma$-trimmed Anderson models with nontrivial background potentials. We are not aware of any previous results on either Wegner estimates or localization for this class of models.

1.2.2. The ground state energy. A trimmed Anderson model $H_{\omega, \lambda}$ is a $K \mathbb{Z}^{d}$ ergodic random Schrödinger operator if and only if $\Gamma=\Gamma+\zeta$ for all $\zeta \in K Z^{d}$, $V^{(0)}$ is a periodic potential with period $K$, and $\mu_{\zeta}=\mu$ for all $\zeta \in \Gamma$. In this case its spectrum $\sigma\left(H_{\boldsymbol{\omega}, \lambda}\right)$ is not random, i.e., it is the same with probability one. In particular, requiring $0=\inf \operatorname{supp} \mu$, we get

$$
E_{\emptyset}\left(H_{\omega, \lambda}\right)=E_{\emptyset}\left(H_{0}\right) \text { with probability one. }
$$

Since a trimmed Anderson model $H_{\omega, \lambda}$ is not, in general, an ergodic random operator, its spectrum $\sigma\left(H_{\omega, \lambda}\right)$ is a random set. We have $E_{\emptyset}\left(H_{\omega, \lambda}\right) \geq E_{\emptyset}\left(H_{0}\right)$ for all $\omega \in[0, M]^{\Gamma}$ and $\lambda>0$. But even after imposing $\mu_{\zeta}=\mu$ for all $\zeta \in \Gamma$ with $0=\inf \operatorname{supp} \mu$ we cannot guarantee (1.9). For example, take $V^{(0)}=-6 d \chi_{\zeta_{0}}$ for some $\zeta_{0} \in \Gamma, \mu$ uniformly distributed on $[0,1]$, and $\lambda>6 d$. Then $E_{\emptyset}\left(H_{0}\right) \leq$ $\left\langle\delta_{\zeta_{0}}, H_{0} \delta_{\zeta_{0}}\right\rangle=-4 d$, but we clearly have $\mathbb{P}\left\{E_{\emptyset}\left(H_{\omega, \lambda}\right) \geq 0\right\}>0$, so (1.9) is not true. But if in addition we require $V^{(0)}$ to be a periodic potential with period $K$, it follows that (1.9) holds by comparison with the ergodic random operator we obtain by removing the $\Gamma$-trimming, that is, replacing $\Gamma$ by $\mathbb{Z}^{d}$. Actually, (1.9) holds in a broader context as the following proposition will show. (See also [31].)

Given a Schrödinger operator $H$ on $\ell^{2}\left(\mathbb{Z}^{d}\right)$, we define finite volume operators $H^{(\Lambda)}=H_{\Lambda^{c}}$, i.e., the restriction $\chi_{\Lambda} H \chi_{\Lambda}$ of $H$ to $\ell^{2}(\Lambda)$, where $\Lambda=\Lambda_{L}(x)$ is a finite box. In particular, given a trimmed Anderson model $H_{\omega, \lambda}$, we define finite volume random operators $H_{\omega, \lambda}^{(\Lambda)}$. We also set $S_{\Lambda}(t):=\max _{\zeta \in \Gamma \cap \Lambda} S_{\mu_{\zeta}}(t)$ for $t \geq 0$, where $S_{\mu}(t):=\sup _{a \in \mathbb{R}} \mu([a, a+t])$ denotes the concentration function of the probability measure $\mu$, and let $S(t):=\sup _{\zeta \in \Gamma} S_{\mu_{\zeta}}(t)$ for $t \geq 0$

Proposition 1.5. Let $H_{\omega, \lambda}$ be a $\Gamma$-trimmed Anderson model with $\mu_{\zeta}=\mu$ for all $\zeta \in \Gamma$ with $0=\inf \operatorname{supp} \mu$. Suppose for any $\varepsilon>0$ there is $L=L(\varepsilon)>0$ such that

$$
\left|\left\{x \in \mathbb{Z}^{d}: E_{\emptyset}\left(H_{0}^{\left(\Lambda_{L}(x)\right)}\right) \leq E_{\emptyset}\left(H_{0}\right)+\varepsilon\right\}\right|=\infty .
$$

Then $E_{\emptyset}\left(H_{0}\right)$ is in the essential spectrum of $H_{0}$ and $E_{\emptyset}\left(H_{\omega, \lambda}\right)=E_{\emptyset}\left(H_{0}\right)$ with probability one.

The proof is given in Section 3.1.

1.2.3. Wegner estimates and localization. We prove Wegner estimates and localization for $\Gamma$-trimmed Anderson models in intervals of the kind $\left[E_{\emptyset}\left(H_{0}\right), E_{1}\right] \subset$ 
$\left[E_{\emptyset}\left(H_{0}\right), E_{\Gamma}\left(H_{0}\right)\right)$. Note that we have (1.9), and hence almost sure existence of the spectrum in these intervals, that is,

$$
\mathbb{P}\left\{\sigma\left(H_{\omega, \lambda}\right) \cap\left[E_{\emptyset}\left(H_{0}\right), E_{1}\right] \neq \emptyset\right\}=1, \quad E_{1}>E_{\emptyset}\left(H_{0}\right),
$$

for the class of (generally) non-ergodic trimmed Anderson models given in Proposition 1.5 .

Theorem 1.6. Let $H_{\omega, \lambda}$ be a $\Gamma$-trimmed Anderson model. Given an energy $E_{1} \in$ $\left(E_{\emptyset}\left(H_{0}\right), E_{\Gamma}\left(H_{0}\right)\right)$, set

$$
\kappa=\kappa\left(H_{0}, \Gamma, E_{1}\right)=\sup _{\substack{s>0 \\ E_{\Gamma}\left(H_{0}, s\right)>E_{1}}} \frac{E_{\Gamma}\left(H_{0}, s\right)-E_{1}}{s}>0 .
$$

Then for every box $\Lambda=\Lambda_{L}\left(x_{0}\right)$ with $x_{0} \in \mathbb{Z}^{d}$ and $L>0$ we have

$$
\chi_{\left(-\infty, E_{1}\right]}\left(H_{\omega, \lambda}^{(\Lambda)}\right) \chi_{\Gamma \cap \Lambda} \chi_{\left(-\infty, E_{1}\right]}\left(H_{\omega, \lambda}^{(\Lambda)}\right) \geq \kappa \chi_{\left(-\infty, E_{1}\right]}\left(H_{\omega, \lambda}^{(\Lambda)}\right),
$$

for $\omega \in[0, M]^{\Gamma}$, and for any closed interval $I \subset\left(-\infty, E_{1}\right]$ we have

$$
\mathbb{E}\left\{\operatorname{tr} \chi_{I}\left(H_{\omega, \lambda}^{(\Lambda)}\right)\right\} \leq 8 \kappa^{-1} S_{\Lambda}\left(\lambda^{-1}|I|\right)|\Gamma \cap \Lambda|
$$

Remark 1.7. It follows from (1.3) (and its proof) that

$$
\kappa \geq \frac{Q}{2 d K+1}\left((1+Z) Y_{d, V^{(0)}}\right)^{-2 d K}
$$

where

$$
Z=\frac{2 K d+1}{2 K d}\left(\left(1-\left(\left(E_{1}-E_{\emptyset}\left(H_{0}\right)\right) Q^{-1}(2 d K-1) Y_{d, V^{(0)}}^{2 d K-1}\right)\right)^{-\frac{1}{2 d K-1}}-1\right) .
$$

(See (3.2)-(3.6) for the derivation of (1.14).)

Theorem 1.6 is proved in Section 3.2.

The Wegner type estimate (1.13) allows us to establish localization for $\Gamma$-trimmed Anderson models at the bottom of the spectrum. By complete localization on an interval $I$ we mean that for all $E \in I$ there exists $\delta(E)>0$ such that we can perform the bootstrap multiscale analysis on the interval $(E-\delta(E), E+\delta(E))$, obtaining Anderson and dynamical localization; see [17], [18], and [19]. (Note that by this definition we always have complete localization in $\left(-\infty, E_{\emptyset}\left(H_{0}\right)\right)$.)

The following theorem show that we always have localization below $E_{\emptyset}\left(H_{0}\right)$ at high disorder.

Theorem 1.8. Let $H_{\omega, \lambda}$ be a $\Gamma$-trimmed Anderson model, and suppose $S(t) \leq$ $C t^{\theta}$ for all $t \geq 0$, where $\theta \in(0,1]$ and $C$ is a constant. Then, given $E_{1} \in$ $\left(E_{\emptyset}\left(H_{0}\right), E_{\Gamma}\left(H_{0}\right)\right)$, there exists $\lambda\left(E_{1}\right)<\infty$ such that $H_{\omega, \lambda}$ exhibits complete localization on the interval $\left(-\infty, E_{1}\right)$ for all $\lambda \geq \lambda\left(E_{1}\right)$. 
Theorem 1.8 is proved exactly as [24], Theorem 1.7, using the Wegner estimate (1.13), so we omit the proof.

We also establish localization in an interval at the bottom of the spectrum for fixed disorder.

Theorem 1.9. Let $H_{\omega, \lambda}$ be a $\Gamma$-trimmed Anderson model, and suppose $S(t) \leq C t^{\theta}$ for all $t \geq 0$, where $\theta \in(0,1]$ and $C$ is a constant. Assume in addition that one of the following hypotheses hold.

(i) $H_{\omega, \lambda}$ is an ergodic $\Gamma$ - trimmed Anderson model.

(ii) There is no background potential, that is, $V^{(0)}=0$.

(iii) The exponent $\theta$ satisfies $\theta>\frac{d}{2}$.

Then for all $\lambda>0$ there exists $E_{\lambda}>E_{\emptyset}\left(H_{0}\right)$ such that $H_{\omega, \lambda}$ exhibits complete localization on the interval $\left(-\infty, E_{\lambda}\right)$.

The proof of this theorem is standard once we have the Wegner estimate (1.13). (Thus we will have $E_{\lambda}<E_{\Gamma}\left(H_{0}\right)$.) The necessary input for starting the multiscale analysis can be verified as follows.

(i) If $H_{\boldsymbol{\omega}, \lambda}$ is ergodic, it has Lifshitz tails $[29,26]$ (the proofs apply also to the discrete case), and we proceed as in [21], Proposition 4.3.

(ii) If $V^{(0)}=0$, we proceed as in [21], Remark 4.4; the argument can be adapted to the discrete case as noted in [30], Theorem 1.2.6, and [31].

(iii) If $\theta>\frac{d}{2}$, we employ the same strategy as in (i), replacing the Lipschitz tails with the "classical tails" given by the condition $\theta>\frac{d}{2}$ as in [14], Proof of Theorem $3^{\prime}$, and [25], Proof of Theorem 3.11.

Acknowledgements. We are grateful to S. Sodin for useful discussions on isoperimetric estimates.

\section{The ground state energy of trimmed Schrödinger operators}

In this section we prove Theorems 1.1 and 1.3. Given $H=-\Delta+V$ on $\ell^{2}\left(\mathbb{Z}^{d}\right)$, where $V$ is a bounded potential, we will use the shorthand notation $E_{\Gamma}=E_{\Gamma}(H)$, $E_{\Gamma}(t)=E_{\Gamma}(H, t), E_{\emptyset}=E_{\emptyset}(H)$.

2.1. Equality of the ground state energies. We start by proving Theorem 1.1.

Proof of Theorem 1.1. We first obtain a simple upper bound on $\delta_{\Gamma}(H)$ (hence on $\delta_{\Gamma}(H, t)$ as well), to be used later on. To this end, note that $E_{\emptyset} \geq \inf _{x \in \mathbb{Z}^{d}} V(x)$, and hence

$$
H_{\Gamma}-E_{\emptyset} \leq-\Delta_{\Gamma}+\operatorname{spr} V \leq 4 d+\operatorname{spr} V .
$$


It follows that

$$
E_{\Gamma}-E_{\emptyset} \leq E_{\Gamma}(-\Delta)+\operatorname{spr} V \leq 2 d+\operatorname{spr} V
$$

where we used $E_{\Gamma}(-\Delta) \leq \inf _{x \in \Gamma^{c}}\left\langle\delta_{x},-\Delta \delta_{x}\right\rangle=2 d$, that is,

$$
\delta_{\Gamma}(H) \leq \delta_{\Gamma}(-\Delta)+\operatorname{spr}(V) \leq 2 d+\operatorname{spr}(V) .
$$

Suppose $E_{\Gamma}>E_{\emptyset}$, since otherwise there is nothing to prove. By replacing $H$ by $H-E_{\emptyset}$, we may assume $E_{\emptyset}=0$, so $\delta_{\Gamma}(H)=E_{\Gamma}$ and $\delta_{\Gamma}(H, t)=E_{\Gamma}(t)$.

Let $v>0$. Then $H+v \geq v$ (recall $\left.E_{\emptyset}=0\right)$, so $(H+v)^{-1} \leq \frac{1}{v}$. It follows that on $\ell^{2}(\Gamma)$ we have

$$
S_{v}=H_{\Gamma^{\mathrm{c}}}+v-u\left(H_{\Gamma}+v\right)^{-1} u^{*} \geq v, \quad u=\chi_{\Gamma} \Delta \chi_{\Gamma^{\mathrm{c}}},
$$

since $S_{v}$ is the the Schur complement of $H_{\Gamma}+v$, and we have

$$
S_{v}^{-1}=\chi_{\Gamma}(H+v)^{-1} \chi_{\Gamma} \leq \frac{1}{v} .
$$

In particular, we conclude that

$$
H_{\Gamma^{\mathrm{c}}} \geq u\left(H_{\Gamma}+v\right)^{-1} u^{*} \text { for all } v>0
$$

By hypothesis $E_{\Gamma}>0$, so we take $\eta \in\left(0, E_{\Gamma}\right)$. Note that for all $v>0$ we have

$$
\left(H_{\Gamma}-\eta\right)^{-1} \leq\left(1+\frac{\eta+v}{E_{\Gamma}-\eta}\right)\left(H_{\Gamma}+v\right)^{-1}
$$

We now consider the Schur complement $S_{-\eta}(t)$ of $\left(H_{\Gamma}(t)\right)_{\Gamma}-\eta$, and use (2.3) and (2.1), getting

$$
\begin{aligned}
S_{-\eta}(t) & =H_{\Gamma^{\mathrm{c}}}+t-\eta-u\left(H_{\Gamma}-\eta\right)^{-1} u^{*} \\
& \geq H_{\Gamma^{\mathrm{c}}}+t-\eta-\left(1+\frac{\eta+v}{E_{\Gamma}-\eta}\right) u\left(H_{\Gamma}+v\right)^{-1} u^{*} \\
& \geq H_{\Gamma^{\mathrm{c}}}+t-\eta-\left(1+\frac{\eta+v}{E_{\Gamma}-\eta}\right) H_{\Gamma^{\mathrm{c}}} \\
& \geq t-\eta-\frac{\eta+v}{E_{\Gamma}-\eta}(4 d+\operatorname{spr}(V)) .
\end{aligned}
$$

Since $v>0$ is arbitrary, we obtain

$$
S_{-\eta}(t) \geq t-\eta-\frac{\eta}{E_{\Gamma}-\eta}(4 d+\operatorname{spr}(V)) .
$$


We conclude that

$$
S_{-\eta}(t)>0
$$

if

$$
\eta<\frac{t+4 d+E_{\Gamma}+\operatorname{spr}(V)-\sqrt{\left(t+4 d+E_{\Gamma}+\operatorname{spr}(V)\right)^{2}-4 E_{\Gamma} t}}{2},
$$

so it follows from the Schur complement condition for positive definiteness that

$$
\begin{aligned}
E_{\Gamma}(t) & \geq \frac{t+4 d+E_{\Gamma}+\operatorname{spr}(V)-\sqrt{\left(t+4 d+E_{\Gamma}+\operatorname{spr}(V)\right)^{2}-4 E_{\Gamma} t}}{2} \\
& =\frac{2 E_{\Gamma} t}{t+4 d+E_{\Gamma}+\operatorname{spr}(V)+\sqrt{\left(t+4 d+E_{\Gamma}+\operatorname{spr}(V)\right)^{2}-4 E_{\Gamma} t}} \\
& \geq \frac{E_{\Gamma} t}{t+4 d+E_{\Gamma}+\operatorname{spr}(V)}, \quad t>0,
\end{aligned}
$$

Combining with (2.2) we get

$$
E_{\Gamma}(t) \geq \frac{E_{\Gamma} t}{t+6 d+2 \operatorname{spr}(V)}, \quad t>0,
$$

which is (1.2), Letting $t \rightarrow \infty$ in (1.2) we get $E_{\Gamma}(\infty) \geq E_{\Gamma}$. Since $E_{\Gamma}(\infty) \leq E_{\Gamma}$, we get $E_{\Gamma}(\infty)=E_{\Gamma}$.

2.2. Lower bounds on the ground state energy for arbitrary potential. Theorem 1.3 for arbitrary bounded potential $V$, namely the lower bounds (1.3)-(1.4), follows from the following theorem.

We recall $Y_{d, V}=2 d+1+\operatorname{spr}(V)$ for a bounded potential $V$.

Theorem 2.1. Let $\Gamma \subsetneq \mathbb{Z}^{d}$ be $(K, Q)$-relatively dense, and let $H=-\Delta+V$ on $\ell^{2}\left(\mathbb{Z}^{d}\right)$, where $V$ is a bounded potential. Then

$$
\delta_{\Gamma}(H, t) \geq \frac{Q}{2 d K-1}\left(\frac{1}{Y_{d, V}^{2 d K-1}}-\frac{1}{\left(Y_{d, V}+t\right)^{2 d K-1}}\right), \quad t \geq 0 .
$$

As a consequence, we get

$$
\delta_{\Gamma}(H) \geq \frac{Q}{(2 d K-1) Y_{d, V}^{2 d K-1}}>0 .
$$

The proof of the theorem is based on what may be called a quantitative unique continuation principle for ground states, given in the following lemma. 
Given a nonempty connected subset $B$ of $\mathbb{Z}^{d}$ and $x, y \in B$, we let $d_{B}(x, y)$ denote the graph distance between $x$ and $y$ in $B$, i.,e., the minimal length of a path in $B$ connecting $x$ and $y$. We set diam $B=\max _{x, y \in B} d_{B}(x, y)$, the diameter of $B$ in the graph theory sense. Note that we always have $d_{B}(x, y) \geq\|x-y\|_{1}$, and $d_{B}(x, y)=\|x-y\|_{1}$ for all $x, y \in B$ if $B=\mathbb{Z}^{d}$ or $B=\Lambda_{L}\left(x_{0}\right)$. In particular, we have diam $\Lambda_{L}\left(x_{0}\right) \leq d L$.

Lemma 2.2. Let $H=-\Delta+V$ on $\ell^{2}\left(\mathbb{Z}^{d}\right)$, where $V$ is a bounded potential. Let $\Lambda=\Lambda_{L}\left(x_{0}\right)$ be a box in $\mathbb{Z}^{d}$. Then $E^{(\Lambda)}=\inf \sigma\left(H_{\Lambda}\right)$ is a simple eigenvalue, and there exists a unique strictly positive ground state $\psi_{g}^{(\Lambda)}$, i.e., there exists a unique $\psi_{g}^{(\Lambda)} \in \ell^{2}(\Lambda)$ such that $H_{\Lambda} \psi_{g}^{(\Lambda)}=E^{(\Lambda)} \psi_{g}^{(\Lambda)},\left\|\psi_{g}^{(\Lambda)}\right\|=1$, and $\psi_{g}^{(\Lambda)}(x)>0$ for all $x \in \Lambda$. Moreover, for all $x \in \Lambda$ and $m \in \mathbb{N}$ we have

$$
\psi_{g}^{(\Lambda)}(x) \geq Y_{d, V}^{-m} \sum_{\substack{y \in \Lambda \\\|x-y\|_{1} \leq m}} \psi_{g}^{(\Lambda)}(y) .
$$

We also get a uniform lower bound:

$$
\psi_{g}^{(\Lambda)}(x) \geq Y_{d, V}^{-d L}, \quad x \in \Lambda
$$

Proof. Without loss of generality we assume $0=\inf _{x \in \mathbb{Z}^{d}} V(x)$, so $0 \leq V \leq V_{\infty}=$ $\operatorname{spr}(V)$ and $E^{(\Lambda)} \geq 0$.

Note that $\ell^{2}(\Lambda)$ is a finite-dimensional Hilbert space. The existence of the unique strictly positive ground state follows from the Perron-Frobenius Theorem. This can be seen as follows. The self-adjoint operator $T=2 d+1+V_{\infty}-H_{\Lambda}$ on $\ell^{2}(\Lambda)$ is positivity preserving, i.e., $\left\langle\delta_{x}, T \delta_{y}\right\rangle \geq 0$ for all $x, y \in \Lambda$. Moreover,

$$
\left\langle\delta_{x}, T^{m} \delta_{y}\right\rangle \geq 1 \quad \text { for } m \geq\|x-y\|_{1}, x, y \in \Lambda \text {. }
$$

In particular, recalling diam $\Lambda \leq d L$, we have

$$
\left\langle\delta_{x}, T^{d L} \delta_{y}\right\rangle \geq 1, \quad x, y \in \Lambda .
$$

It follows from the Perron-Frobenius Theorem that $\lambda_{\max }=\max \sigma(T)$ is a simple eigenvalue, and there exists a unique $\psi_{g}^{(\Lambda)} \in \ell^{2}(\Lambda)$ such that $T \psi_{g}^{(\Lambda)}=\lambda_{\max } \psi_{g}^{(\Lambda)}$, $\left\|\psi_{g}^{(\Lambda)}\right\|=1$, and $\psi_{g}^{(\Lambda)}(x)>0$ for all $x \in \Lambda$. Clearly, $H_{\Lambda} \psi_{g}^{(\Lambda)}=E^{(\Lambda)} \psi_{g}^{(\Lambda)}$ and

$$
\lambda_{\max }=2 d+1+V_{\infty}-E^{(\Lambda)} \leq 2 d+1+V_{\infty}=Y_{d, V} .
$$

Moreover, since $T \psi_{g}^{(\Lambda)}=\lambda_{\max } \psi_{g}^{(\Lambda)}$ and $\psi_{g}^{(\Lambda)}(x)>0$ for all $x \in \Lambda$, we have for all $x \in \Lambda$ and $m \in \mathbb{N}\left(\psi_{g}=\psi_{g}^{(\Lambda)}\right)$

$$
\psi_{g}(x) \geq \lambda_{\max }^{-m} \sum_{y \in \Lambda ;} \sum_{\|x-y\|_{1} \leq m} \psi_{g}(y),
$$

which yields (2.8).

To get (2.9), just notice that $1=\left\|\psi_{g}\right\|_{2} \leq\left\|\psi_{g}\right\|_{1}=\sum_{y \in \Lambda} \psi_{g}(y)$. 
Proof of Theorem 2.1. Given $\zeta \in K \mathbb{Z}^{d}$, fix $\Gamma_{\zeta} \subset \Gamma \cap \Lambda_{K}^{(0)}(\zeta)$ such that $\left\|\Gamma_{\zeta}\right\|=Q$.

Let $R=K J$ where $J=1,3,5, \ldots$ and consider $\Lambda=\Lambda_{R}=\Lambda_{R}(0)$. Then, by Lemma 2.2, for all $t \geq 0$ we have that $E^{(\Lambda)}(t)=\inf \sigma\left(H_{\Gamma}(t)\right)$ is a simple isolated eigenvalue with eigenvector $\psi_{g, t}^{(\Lambda)}$ as in Lemma 2.2, so it follows that the orthogonal projection $P_{g}(t)=\left\langle\psi_{g, t}^{(\Lambda)}, \cdot \psi_{g, t}^{(\Lambda)}\right\rangle \psi_{g, t}^{(\Lambda)}$ is differentiable in $t$, and

$$
\begin{aligned}
\frac{\mathrm{d}}{\mathrm{d} t} E^{(\Lambda)}(t) & =\frac{\mathrm{d}}{\mathrm{d} t} \operatorname{tr} P_{g}(t) H(t) \\
& =\operatorname{tr} P_{g}(t) \dot{H}(t)+\operatorname{tr} \dot{P}_{g}(t) H(t) \\
& =\operatorname{tr} P_{g}(t) \dot{H}(t) \\
& =\operatorname{tr} P_{g}(t) \chi_{\Gamma} \\
& \geq \sum_{\zeta \in K \mathbb{Z}^{d} \cap \Lambda}\left\langle\psi_{g, t}^{(\Lambda)}, \chi_{\Gamma_{\zeta}} \psi_{g, t}^{(\Lambda)}\right\rangle \\
& =\sum_{\zeta \in K \mathbb{Z}^{d} \cap \Lambda} \sum_{x \in \Gamma_{\zeta}}\left(\psi_{g, t}^{(\Lambda)}(x)\right)^{2},
\end{aligned}
$$

where on the second line we have used $\dot{P}_{g}=P_{g} \dot{P}_{g}\left(1-P_{g}\right)+\left(1-P_{g}\right) \dot{P}_{g} P_{g}$, cyclicity of the trace, and $P_{g} H\left(1-P_{g}\right)=0$.

If $x \in \Gamma_{\zeta}$, it follows from (2.8) that

$$
\psi_{g, t}^{(\Lambda)}(x) \geq\left(Y_{d, V}+t\right)^{-d K} \sum_{y \in \chi_{\Lambda_{K}(\zeta)}} \psi_{g, t}^{(\Lambda)}(y),
$$

and hence

$$
\left(\psi_{g, t}^{(\Lambda)}(x)\right)^{2} \geq\left(Y_{d, V}+t\right)^{-2 d K} \sum_{y \in \chi_{\Lambda_{K}(\zeta)}}\left(\psi_{g, t}^{(\Lambda)}(y)\right)^{2} .
$$

Combining (2.10) and (2.11) we get

$$
\begin{aligned}
\frac{\mathrm{d}}{\mathrm{d} t} E^{(\Lambda)}(t) & \geq Q\left(Y_{d, V}+t\right)^{-2 d K} \sum_{\zeta \in K \mathbb{Z}^{d} \cap \Lambda} \sum_{y \in \chi_{\Lambda_{K}(\zeta)}}\left(\psi_{g, t}^{(\Lambda)}(y)\right)^{2} \\
& =Q\left(Y_{d, V}+t\right)^{-2 d K} .
\end{aligned}
$$

Thus

$$
\begin{aligned}
E^{(\Lambda)}(t)-E^{(\Lambda)}(0) & \geq Q \int_{0}^{t} \mathrm{~d} s\left(Y_{d, V}+s\right)^{-2 d K} \\
& =\frac{Q}{2 d K-1}\left(\frac{1}{Y_{d, V}^{2 d K-1}}-\frac{1}{\left(Y_{d, V}+t\right)^{2 d K-1}}\right) .
\end{aligned}
$$

To conclude the proof of the theorem, just note that $E_{\Gamma}(t)=\lim _{R \rightarrow \infty} E\left(\Lambda_{R}\right)(t)$ for all $t \geq 0$. 
2.3. Cheeger's inequality for the ground state energy. Theorem 1.3 for $H=-\Delta$, namely the lower bound (1.5), follows from the following theorem.

Theorem 2.3. Let $\Gamma \subsetneq \mathbb{Z}^{d}$ be $(K, Q)$-relatively dense. Then

$$
E_{\Gamma}(-\Delta) \geq \frac{1}{4 d K_{*}^{2 d}} \text {. }
$$

In addition,

$$
E_{\Gamma}(-\Delta, t) \geq \frac{1}{(6 d-1) K_{*}^{2 d}}, \quad t \geq 2 d-1 .
$$

Remark 2.4. For $H=-\Delta$ the estimate (2.13) in Theorem 2.3 is better than the corresponding estimate from Theorem 2.1. Note that (2.14) only holds for $t \geq 2 d-1$, giving a lower bound independent of $t$. We can get an estimate for all $t \geq 0$ by combining (2.13) and (2.4), getting

$$
E_{\Gamma}(t) \geq \frac{t}{4 d K_{*}^{2 d}(t+4 d)+1} .
$$

This estimate is better than (2.14) for sufficiently large $t$.

Given $A \subset \mathbb{Z}^{d}$, let

- $\partial A=\left\{(x, y) \in A \times A^{\mathrm{c}} ;\|x-y\|=1\right\}$,

- $\partial_{-} A=\left\{x \in A ;(x, y) \in \partial A\right.$ for some $\left.y \in A^{\mathrm{c}}\right\}$,

- $\partial_{+} A=\left\{y \in A^{\mathrm{c}} ;(x, y) \in \partial A\right.$ for some $\left.x \in A\right\}$;

- given $x \in Z^{d}$, set

$$
\eta_{A}(x)=\left|\left\{y \in \mathbb{Z}^{d} ;(x, y) \in \partial A\right\}\right| \in\{0,1,2, \ldots, 2 d\},
$$

so $\partial_{-} A=\left\{x \in \mathbb{Z}^{d} ; \eta_{A}(x) \geq 1\right\}$.

Note that

$$
\left\langle\chi_{A},(-\Delta) \chi_{A}\right\rangle=|\partial A|=\sum_{x \in \mathbb{Z}^{d}} \eta_{A}(x)=\sum_{x \in \partial_{-} A} \eta_{A}(x) .
$$

Lemma 2.5. Let $\Gamma \subsetneq \mathbb{Z}^{d}$ be $(K, Q)$-relatively dense. Then for all $A \subset \mathbb{Z}^{d} \backslash \Gamma$ we have

$$
\left\langle\chi_{A},(-\Delta) \chi_{A}\right\rangle=|\partial A| \geq K_{*}^{-d}|A| \text {. }
$$

Proof. Let $A \subset \mathbb{Z}^{d} \backslash \Gamma_{K}$, set $A_{\zeta}=A \cap \Lambda_{K}(\zeta)$ for $\zeta \in K \mathbb{Z}^{d}$, and let $N_{A}=\mid\{\zeta \in$ $\left.K \mathbb{Z}^{d} ; A_{\zeta} \neq \emptyset\right\} \mid$. Then

$$
|A| \leq K_{*}^{d} N_{A}
$$

On the other hand, $A_{\zeta} \neq \varnothing$ implies $\partial A \cap\left(\Lambda_{K}(\zeta) \times \Lambda_{K}^{(0)}(\zeta)\right) \neq \emptyset$ since $\Gamma_{K} \cap \Lambda_{K}^{(0)}(\zeta) \neq$ $\emptyset$. We conclude that $N_{A} \leq|\partial A|$, so (2.16) follows from (2.17). 
Let $H=-\Delta$ and fix $\Gamma \subsetneq \mathbb{Z}^{d}$ be $(K, Q)$-relatively dense. Following [28], we define the Cheeger constants (note $\left\langle\chi_{A}, \chi_{A}\right\rangle=|A|$ )

$$
\beta(\Gamma)=\inf _{A \subset \mathbb{Z}^{d} \backslash \Gamma ; 1 \leq|A|<\infty} \beta_{A}(\Gamma), \quad \text { where } \beta_{A}(\Gamma)=\frac{\left\langle\chi_{A},\left(-\Delta_{\Gamma}\right) \chi_{A}\right\rangle}{|A|},
$$

and

$$
\beta(t)=\inf _{A \subset \mathbb{Z}^{d} ; 1 \leq|A|<\infty} \beta_{A}(t), \quad \text { where } \beta_{A}(t)=\frac{\left\langle\chi_{A}, H(t) \chi_{A}\right\rangle}{|A|}, t \geq 0 .
$$

Clearly $\beta(\Gamma) \geq E_{\Gamma}$ and $\beta(t) \geq E(t)$ for all $t \geq 0$.

Lemma 2.6. We have

$$
K_{*}^{-d} \leq \beta(\Gamma) \leq 2 d .
$$

Moreover, $\beta(t)$ is a nondecreasing function of $t \geq 0$, and

$$
\beta(t) \geq \beta^{(1)}(\Gamma) \geq K_{*}^{-d}, \quad t \geq 2 d-1,
$$

where $\beta^{(1)}(\Gamma)=\min \{\beta(\Gamma), 1\}$.

Proof. Given $A \subset \mathbb{Z}^{d} \backslash \Gamma,|A| \geq 1$, it follows from Lemma 2.5, that

$$
\beta_{A}(t)=\beta_{A}(\Gamma)=\frac{\left\langle\chi_{A},(-\Delta) \chi_{A}\right\rangle}{|A|} \geq K_{*}^{-d}, \quad t \geq 0 .
$$

It follows that $\beta(\Gamma) \geq K_{*}^{-d}$. On the other hand, there exists $y_{0} \in \mathbb{Z}^{d} \backslash \Gamma$, since $\Gamma \subsetneq \mathbb{Z}^{d}$, and we have

$$
\beta(\Gamma) \leq \beta_{\left\{y_{0}\right\}}(\Gamma) \leq 2 d .
$$

Let $A \subset \mathbb{Z}^{d} ; 1 \leq|A|<\infty$. Suppose $x \in A \cap \Gamma, A_{x}=A \backslash\{x\}$, and assume $\left|A_{x}\right| \geq 1$. Then $|A|=\left|A_{x}\right|+1$ and

$$
\left\langle\chi_{A}, H(t) \chi_{A}\right\rangle \geq\left\langle\chi_{A_{x}}, H(t) \chi_{A_{x}}\right\rangle-2 d+t,
$$

so, if $t \geq 2 d-1$,

$$
\beta_{A_{x}}(t) \leq \frac{\left\langle\chi_{A}, H(t) \chi_{A}\right\rangle-1}{|A|-1} \leq \frac{\left\langle\chi_{A}, H(t) \chi_{A}\right\rangle}{|A|}=\beta_{A}(t),
$$

assuming $\left\langle\chi_{A}, H(t) \chi_{A}\right\rangle \leq|A|$, i.e., $\beta_{A}(t) \leq 1$. If $|A \backslash \Gamma| \geq 1$, repeating this procedure until we removed all points of $\Gamma$ from the set $A$ we obtain

$$
\beta_{A}(t) \geq \beta_{A \backslash \Gamma}(t)=\beta_{A \backslash \Gamma}(\Gamma) \geq \beta(\Gamma) .
$$

If $A \subset \Gamma,|A| \geq 1$, we pick $x_{0} \in A$, so we get

$$
\beta_{A}(t) \geq \beta_{\left\{x_{0}\right\}}(t)=2 d+t \geq 2 d \geq \beta(\Gamma) .
$$

We thus conclude that for all $t \geq 2 d-1$ we have $\beta_{A}(t) \geq \beta^{(1)}(\Gamma)$ for all $A \subset \mathbb{Z}^{d}$ such that $1 \leq|A|<\infty$. The lemma follows. 
Theorem 2.3 follows from the following theorem.

Theorem 2.7. Let $\Gamma \subsetneq \mathbb{Z}^{d}$ be $(K, Q)$-relatively dense. Then

$$
E_{\Gamma}(-\Delta) \geq \frac{(\beta(\Gamma))^{2}}{4 d} \geq \frac{1}{4 d K_{*}^{2 d}}
$$

In addition,

$$
E_{\Gamma}(-\Delta, t) \geq \frac{\left(\beta^{(1)}(\Gamma)\right)^{2}}{6 d-1} \geq \frac{1}{(6 d-1) K_{*}^{2 d}}, \quad t \geq 2 d-1 .
$$

Proof. We write

$$
\begin{aligned}
H(t) & =H_{\Gamma}(t)=-\Delta+t \chi_{\Gamma} \\
E_{\Gamma} & =E_{\Gamma}(-\Delta), \\
E(t) & =E_{\Gamma}(-\Delta, t) .
\end{aligned}
$$

We prove (2.20) first. Following [28], we introduce $\widehat{\mathbb{Z}^{d}}=\mathbb{Z}^{d} \cup\{\infty\}$, and for $t>0$ define the self-adjoint bounded operator $\widehat{H(t)}$ on $\ell^{2}\left(\widehat{\mathbb{Z}^{d}}\right)$ by

$$
\widehat{H(t)} \varphi(x)=\sum_{y \in \widehat{\mathbb{Z}}^{d}} \kappa(x, y)(\varphi(x)-\varphi(y)),
$$

where

(i) $\kappa(x, y)=1$ for $x, y \in \mathbb{Z}^{d},|x-y|=1$,

(ii) $\kappa(x, y)=0$ for $x, y \in \mathbb{Z}^{d},|x-y| \neq 1$,

(iii) $\kappa(x, \infty)=\kappa(\infty, x)=t \chi_{\Gamma}(x)$ for $x \in \mathbb{Z}^{d}$,

(iv) $\kappa(\infty, \infty)=0$.

Given $\varphi \in \ell^{2}\left(\mathbb{Z}^{d}\right)$, we extend it to $\hat{\varphi} \in \ell^{2}\left(\widehat{\mathbb{Z}^{d}}\right)$ by setting $\hat{\varphi}(\infty)=0$. It follows that $\widehat{H(t) \varphi}=\widehat{H(t)} \hat{\varphi}$, and we have

$$
\langle\varphi, H(t) \varphi\rangle_{\ell^{2}\left(\mathbb{Z}^{d}\right)}=\langle\widehat{\varphi}, \widehat{H(t)} \hat{\varphi}\rangle_{\ell^{2}\left(\widehat{\mathbb{Z}^{d}}\right)}=\frac{1}{2} \sum_{x, y \in \widehat{\mathbb{Z}^{d}}} \kappa(x, y)|\hat{\varphi}(x)-\hat{\varphi}(y)|^{2} .
$$

Note that

$$
\langle\varphi, H(t) \varphi\rangle=\langle\varphi,(-\Delta) \varphi\rangle+t\left\|\chi_{\Gamma} \varphi\right\|^{2}
$$

so

$$
\begin{aligned}
E(t) & =\inf \left\{\langle\varphi, H(t) \varphi\rangle: \varphi \in \ell^{2}\left(\mathbb{Z}^{d}\right),\|\varphi\|=1\right\} \\
& =\inf \left\{\langle\varphi, H(t) \varphi\rangle: \varphi \in \ell^{2}\left(\mathbb{Z}^{d} ; \mathbb{R}\right),\|\varphi\|=1,|\operatorname{supp} \varphi|<\infty\right\}
\end{aligned}
$$


Now let $\varphi$ be a real-valued function on $\mathbb{Z}^{d}$ with finite support. We have, using the Cauchy-Schwarz inequality,

$$
\begin{aligned}
2\langle\varphi, H(t) \varphi\rangle_{\ell^{2}\left(\mathbb{Z}^{d}\right)} & =\sum_{x, y \in \widehat{\mathbb{Z}^{d}}} \kappa(x, y)(\hat{\varphi}(x)-\hat{\varphi}(y))^{2} \\
& \geq \frac{\left(\sum_{x, y \in \widehat{\mathbb{Z}^{d}}} \kappa(x, y)\left|\hat{\varphi}(x)^{2}-\hat{\varphi}(y)^{2}\right|\right)^{2}}{\sum_{x, y \in \widehat{\mathbb{Z}}^{d}} \kappa(x, y)(\hat{\varphi}(x)+\hat{\varphi}(y))^{2}} .
\end{aligned}
$$

For the denominator, we have

$$
\begin{aligned}
& \sum_{x, y \in \widehat{\mathbb{Z}}^{d}} \kappa(x, y)(\hat{\varphi}(x)+\hat{\varphi}(y))^{2} \\
& =\sum_{x, y \in \mathbb{Z}^{d} ;|x-y|=1}(\varphi(x)+\varphi(y))^{2}+2 t\left\langle\varphi, \chi_{\Gamma} \varphi\right\rangle \\
& \leq \sum_{x, y \in \mathbb{Z}^{d} ;|x-y|=1}\left(2 \varphi(x)^{2}+2 \varphi(y)^{2}\right)+2 t\left\langle\varphi, \chi_{\Gamma} \varphi\right\rangle \leq 8 d\|\varphi\|^{2}+2 t\left\|\chi_{\Gamma} \varphi\right\|^{2} .
\end{aligned}
$$

For the numerator, since $\kappa$ is symmetric, we have, setting

$$
A_{s}=\left\{\hat{\varphi}^{2}>s\right\}=\left\{\varphi^{2}>s\right\}
$$

for $s \geq 0$,

$$
\begin{aligned}
& \sum_{x, y \in \widehat{\mathbb{Z}^{d}}} \kappa(x, y)\left|\hat{\varphi}(x)^{2}-\hat{\varphi}(y)^{2}\right| \\
& =2 \sum_{x, y \in \widehat{\mathbb{Z}^{d}}} \kappa(x, y) \chi\left(\left\{\hat{\varphi}(x)^{2}>\hat{\varphi}(y)^{2}\right\}\right)\left|\hat{\varphi}(x)^{2}-\hat{\varphi}(y)^{2}\right| \\
& =2 \int_{0}^{\infty} \mathrm{d} s \sum_{x, y \in \widehat{\mathbb{Z}}^{d}} \kappa(x, y) \chi\left(\left\{\hat{\varphi}(x)^{2}>s \geq \hat{\varphi}(y)^{2}\right\}\right) \\
& =2 \int_{0}^{\infty} \mathrm{d} s \sum_{x, y \in \widehat{\mathbb{Z}^{d}}} \kappa(x, y) \chi_{A_{s}}(x)\left(\chi_{A_{s}}(x)-\chi_{A_{s}}(y)\right) \\
& =2 \int_{0}^{\infty} \mathrm{d} s\left\langle\chi_{A_{s}}, H(t) \chi_{A_{s}}\right\rangle \\
& \geq 2 \beta(t) \int_{0}^{\infty} \mathrm{d} s\left|A_{s}\right| \\
& =2 \beta(t)\|\varphi\|^{2} .
\end{aligned}
$$


We conclude that for a real-valued function $\varphi$ on $\mathbb{Z}^{d}$ with finite support and $\|\varphi\|=1$ we have, for all $t \geq 2 d-1$, using Lemma 2.6,

$$
\langle\varphi, H(t) \varphi\rangle \geq \frac{1}{2} \frac{\left(2 \beta(t)\|\varphi\|^{2}\right)^{2}}{8 d\|\varphi\|^{2}+2 t\left\|\chi_{\Gamma} \varphi\right\|^{2}} \geq \frac{(\beta(t))^{2}}{4 d+t} \geq \frac{\left(\beta^{(1)}(\Gamma)\right)^{2}}{4 d+t} .
$$

Thus

$$
E(t) \geq \frac{\left(\beta^{(1)}(\Gamma)\right)^{2}}{4 d+t}, \quad t \geq 2 d-1 .
$$

Since $E(t)$ is nondecreasing in $t$, we get

$$
E(t) \geq \frac{\left(\beta^{(1)}(\Gamma)\right)^{2}}{6 d-1}, \quad t \geq 2 d-1
$$

To prove (2.19), we repeat the above procedure with $-\Delta_{\Gamma}, \mathbb{Z}^{d} \backslash \Gamma, \mathbb{Z}^{d}$ and $\widehat{-\Delta_{\Gamma}}$ instead of $H(t), \mathbb{Z}^{d}, \widehat{\mathbb{Z}^{d}}$ and $\widehat{H(t)}$, where $\widehat{-\Delta_{\Gamma}}=\left(-\Delta_{\Gamma}\right) \oplus 0$ on $\ell^{2}\left(\mathbb{Z}^{d}\right)=$ $\ell^{2}\left(\mathbb{Z}^{d} \backslash \Gamma\right) \oplus \ell^{2}(\Gamma)$, and $\kappa(x, y)=1$ for $x, y \in \mathbb{Z}^{d},|x-y|=1, \kappa(x, y)=0$ for $x, y \in \mathbb{Z}^{d},|x-y| \neq 1$, and, given $\varphi \in \ell^{2}\left(\mathbb{Z}^{d} \backslash \Gamma\right)$, extending it to $\hat{\varphi} \in \ell^{2}\left(\mathbb{Z}^{d}\right)$ by setting $\hat{\varphi}(x)=0$ for $x \in \Gamma$. The proof goes through in exactly the same way, and we get (2.19).

\section{Trimmed Anderson models}

In this section we prove Proposition 1.5 and Theorem 1.6.

\subsection{The ground state energy}

Proof of Proposition 1.5. Let $H_{\omega, \lambda}$ be a $\Gamma$-trimmed Anderson model with $\mu_{\zeta}=\mu$ for all $\zeta \in \Gamma$ with $0=\inf \operatorname{supp} \mu$. To show that $E_{\emptyset}=E_{\emptyset}\left(H_{0}\right) \in \sigma_{\text {ess }}\left(H_{0}\right)$, it suffices to exhibit an orthonormal sequence $\left\{\varphi_{n}\right\}_{n \in \mathbb{N}}$ in $\ell^{2}\left(\mathbb{Z}^{d}\right)$ such that

$$
\left\|\left(H_{0}-E_{\emptyset}\right) \varphi_{n}\right\| \leq 1 / n, \quad n \in \mathbb{N} .
$$

The existence of such a sequence follows from (1.10). Hence $E_{\emptyset} \in \sigma_{\text {ess }}\left(H_{0}\right)$ by Weyl's criterion.

To show that (1.9) holds, for each $\varepsilon>0$ we use (1.10) to construct an orthonormal sequence $\left\{\psi_{n}^{(\varepsilon)}\right\}_{n \in \mathbb{N}}$ in $\ell^{2}\left(\mathbb{Z}^{d}\right)$ such that supp $\psi_{n}^{(\varepsilon)} \subset \Lambda_{L}\left(x_{n}\right)$ with $L=L(\varepsilon)$ for all $n \in N$, with $\left\|x_{n}-x_{m}\right\|_{\infty}>L$ for $n \neq m$, and

$$
\left\|\left(H_{0}-E_{\emptyset}\right) \psi_{n}^{(\varepsilon)}\right\| \leq \varepsilon, \quad n \in \mathbb{N} .
$$


We then have

$$
\begin{aligned}
E_{\emptyset}\left(H_{\boldsymbol{\omega}, \lambda}\right) & \leq\left\langle\psi_{n}^{(\varepsilon)}, H_{\omega, \lambda} \psi_{n}^{(\varepsilon)}\right\rangle \\
& \leq \varepsilon+\sum_{\zeta \in \Gamma \cap \Lambda_{L}\left(x_{n}\right)} \omega_{\zeta}\left|\psi_{n}^{(\varepsilon)}(\zeta)\right|^{2} \text { for all } n \in \mathbb{N}
\end{aligned}
$$

But

$$
\mathbb{P}\left\{\inf _{n \in \mathbb{N}} \max _{\zeta \in \Lambda_{L}\left(x_{n}\right)} \omega_{\zeta}<\varepsilon L^{-d}\right\}=1
$$

from which it follows that

$$
\left.\mathbb{P}\left\{\sigma\left(H_{\boldsymbol{\omega}, \lambda}\right) \cap\left[E_{\emptyset}, E_{\emptyset}+2 \varepsilon\right] \neq \emptyset\right)\right\}=1 .
$$

Since $\varepsilon$ is arbitrary, the result follows.

\subsection{The Wegner estimate}

Proof of Theorem 1.6. Let $H_{\omega, \lambda}$ be a $\Gamma$-trimmed Anderson model, fix $E_{1} \in\left(E_{\emptyset}\left(H_{0}\right)\right.$, $\left.E_{\Gamma}\left(H_{0}\right)\right)$, and let $\kappa=\kappa\left(H_{0}, \Gamma, E_{1}\right)$ be as in (1.11). We clearly have $\kappa>0$. To derive the explicit bound stated in Remark 1.7, namely (1.14), note that the estimate (1.3) yields

$$
E_{\Gamma}\left(H_{0}, s\right)-E_{\emptyset}\left(H_{0}\right) \geq \frac{Q}{(2 d K-1) Y_{d, V^{(0)}}^{2 d K-1}}\left(1-\left(\frac{Y_{d, V^{(0)}}}{Y_{d, V^{(0)}}+s}\right)^{2 d K-1}\right)
$$

for all $s>0$, which implies $E_{\Gamma}\left(H_{0}, s\right)>E_{1}$ for

$$
s>s_{0}=Y_{d, V^{(0)}}\left(\left(1-\left(E_{1}-E_{\emptyset}\left(H_{0}\right)\right) Q^{-1}(2 d K-1) Y_{d, V^{(0)}}^{2 d K-1}\right)^{-\frac{1}{2 d K-1}}-1\right) .
$$

Using (2.12), we get

$$
\kappa \geq \sup _{s>s_{0}} \frac{E_{\Gamma}\left(H_{0}, s\right)-E_{\Gamma}\left(H_{0}, s_{0}\right)}{s} \geq \sup _{s>s_{0}} \frac{s-s_{0}}{s} Q\left(Y_{d, V^{(0)}}+s\right)^{-2 d K} .
$$

The supremum is attained at

$$
s=\frac{2 K d+1}{4 K d}\left(1+\sqrt{1+\frac{8 K d Y_{d, V^{(0)}}}{(2 K d+1)^{2} s_{0}}}\right) s_{0}
$$

to get a simpler lower bound we take $s=s=\frac{2 K d+1}{2 K d} s_{0}$, getting

$$
\kappa \geq \frac{Q}{2 d K+1}\left(Y_{d, V^{(0)}}+\frac{2 K d+1}{2 K d} s_{0}\right)^{-2 d K},
$$

which is (1.14). 
We now proceed as in [24], Proof of Theorem 1.7. Let $\Lambda=\Lambda_{L}\left(x_{0}\right)$ with $x_{0} \in \mathbb{Z}^{d}$ and $L>0$, and note that $\left(H_{0, \Gamma}^{(\Lambda)}(t)=\left(\left(H_{0}\right)_{\Gamma}(t)\right)^{(\Lambda)}\right)$

$$
E_{\Gamma}\left(H_{0}^{\Lambda}, t\right)=E_{\emptyset}\left(H_{0, \Gamma}^{(\Lambda)}(t)\right) \geq E_{\emptyset}\left(H_{0, \Gamma}(t)\right)=E_{\Gamma}\left(H_{0}, t\right),
$$

So

$$
\kappa\left(H_{0}^{\Lambda}, \Gamma, E_{1}\right)=\sup _{s>0 ; E_{\Gamma}\left(H_{0}^{\Lambda}, s\right)>E_{1}} \frac{E_{\Gamma}\left(H_{0}^{\Lambda}, s\right)-E_{1}}{s} \geq \kappa\left(H_{0}, \Gamma, E_{1}\right)=\kappa>0 .
$$

As a consequence, (1.12) follows immediately from [24], Lemma 4.1.

The Wegner estimate (1.13) follows using (1.12). For any closed interval $I \subset$ $\left(-\infty, E_{1}\right]$ we have

$$
\begin{aligned}
\operatorname{tr} \chi_{I}\left(H_{\omega, \lambda}^{(\Lambda)}\right) & \leq \kappa^{-1} \operatorname{tr} \chi_{I}\left(H_{\omega, \lambda}^{(\Lambda)}\right) \chi_{\Gamma \cap \Lambda} \chi_{I}\left(H_{\omega, \lambda}^{(\Lambda)}\right) \\
& =\kappa^{-1} \operatorname{tr} \chi_{\Gamma \cap \Lambda} \chi_{I}\left(H_{\omega, \lambda}^{(\Lambda)}\right) \chi_{\Gamma \cap \Lambda} \\
& =\kappa^{-1} \sum_{\zeta \in \Gamma \cap \Lambda}\left\langle\delta_{\zeta}, \chi_{I}\left(H_{\omega, \lambda}^{(\Lambda)}\right) \delta_{\zeta}\right\rangle .
\end{aligned}
$$

Since by spectral averaging, [10], eq. (3.16) (see also [8], Appendix A),

$$
\int \mathrm{d} \mu_{\zeta}\left(\omega_{\zeta}\right)\left\langle\delta_{\zeta}, \chi_{I}\left(H_{\omega, \lambda}^{(\Lambda)}\right) \delta_{\zeta}\right\rangle \leq 8 S_{\mu_{\zeta}}\left(\lambda^{-1}|I|\right),
$$

we get (1.13).

\section{References}

[1] M. Aizenman, Localization at weak disorder: some elementary bounds. Rev. Math. Phys. 6 (1994), 1163-1182. MR 1301371 Zbl 0843.47039

[2] M. Aizenman and S. Molchanov, Localization at large disorder and extreme energies: an elementary derivation. Comm. Math. Phys. 157 (1993), 245-278. MR 1244867 Zbl 0782.60044

[3] M. Aizenman, J. Schenker, B. Friedrich, and D. Hundertmark, Finite volume fractionalmoment criteria for Anderson localization. Comm. Math. Phys. 224 (2001), 219-253. MR 1868998 Zbl 1038.82038

[4] P. W. Anderson, Absence of diffusion in certain random lattices. Phys. Rev. 109 (1958), 1492-1505.

[5] J. Bourgain and G. Kenig, C. On localization in the continuous Anderson-Bernoulli model in higher dimension. Invent. Math. 161 (2005), 389-426. MR 2180453 Zbl 1084.82005

[6] Z. Cao and A. Elgart, The weak localization for the alloy-type Anderson model on a cubic lattice. J. Stat. Phys. 148 (2012), 1006-1039. MR 2975520 Zbl 1260.82045 
[7] J. Cheeger, A lower bound for the smallest eigenvalue of the Laplacian. In R. C. Gunning Problems in analysis. A symposium in honor of Salomon Bochner, Princeton University, Princeton, N.J., 1-3 April 1969. Princeton University Press, Princeton, N.J., 1970, 195-199. MR 0402831 (collection) MR 0337450 Zbl 0212.44903 Zbl 0208.00301 (collection)

[8] J. M. Combes, F. Germinet, and A. Klein, Generalized eigenvalue-counting estimates for the Anderson model. J. Stat. Phys. 135 (2009) 201-216. MR 2505733 Zbl 1168.82016

[9] J. M. Combes, P. D. Hislop, and F. Klopp, Hölder continuity of the integrated density of states for some random operators at all energies. Int. Math. Res. Not. 2003 (2003), 179-209. MR 1935272 Zbl 1022.47028

[10] J. M. Combes, P. D. Hislop, and F. Klopp, An optimal Wegner estimate and its application to the global continuity of the integrated density of states for random Schrödinger operators. Duke Math. J. 140 (2007), 469-498. MR 2362242 Zbl 1134.81022

[11] F. Delyon, Y. Lévy, and B. Souillard, Anderson localization for multidimensional systems at large disorder or large energy. Comm. Math. Phys. 100 (1985), 463-470. MR 0806247 Zbl 0576.60053

[12] J. Dodziuk, Difference equations, isoperimetric inequality and transience of certain random walks. Trans. Amer. Math. Soc. 284 (1984), 787-794. MR 0743744 Zbl 0512.39001

[13] H. von Dreifus and A. Klein, A new proof of localization in the Anderson tight binding model. Comm. Math. Phys. 124 (1989), 285-299. MR 1012868 Zbl 0698.60051

[14] A. Figotin and A. Klein Localization phenomenon in gaps of the spectrum of random lattice operators. J. Stat. Phys. 75 (1994), 997-1021. MR 1285295 Zbl 0838.47050

[15] J. Fröhlich, F. Martinelli, E. Scoppola, and T. Spencer, Constructive proof of localization in the Anderson tight binding model. Commun. Math. Phys. 101 (1985), 21-46. MR 0814541 Zbl 0573.60096

[16] J. Fröhlich and T. Spencer, Absence of diffusion in the Anderson tight binding model for large disorder or low energy. Comm. Math. Phys. 88 (1983), 151-184. MR 0696803 Zbl 0519.60066

[17] F. Germinet and A. Klein, Bootstrap multiscale analysis and localization in random media. Comm. Math. Phys. 222 (2001), 415-448. MR 1859605 Zbl 0982.82030

[18] F. Germinet and A. Klein, Explicit finite volume criteria for localization in continuous random media and applications. Geom. Funct. Anal. 13 (2003), 1201-1238. MR 2033837 Zbl 1086.82008

[19] F. Germinet and A. Klein, New characterizations of the region of complete localization for random Schrödinger operators. J. Stat. Phys. 122 (2006), 73-94. MR 2203782 Zbl 1127.82031

[20] F. Germinet and A. Klein, Random Schrödinger operators: localization and delocalization, and all that. In V. Sidoravičius (ed.), New Trends in Mathematical Physics. Selected contributions of the XV international congress on mathematical physics, Rio de Janeiro, Brazil, August 5-11, 2006. Springer, Dordrecht, 2009, 371-388. Zbl 1175.81094 Zbl 1170.81006 (collection)

[21] F. Germinet and A. Klein, A comprehensive proof of localization for continuous Anderson models with singular random potentials. J. Eur. Math. Soc. 15 (2013), 53-143. MR 2998830 Zbl 1267.82066 
[22] F. Gesztesy, G. M. Graf, and B. Simon, The ground state energy of Schrödinger operators. Comm. Math. Phys. 150 (1992), 375-384. MR 1194022 Zbl 0784.34061

[23] W. Kirsch, An invitation to random Schröinger operators. In M. Disertori, W. Kirsch, A. Klein, F. Klopp, and V. Rivasseau (eds.), Random Schrödinger operators. Panoramas et Synthèses 25. Société Mathématique de France, Paris, 2008, 1-119. MR 2509110 MR 2516524 (collection) Zbl 1162.82004 Zbl 1151.35002 (collection)

[24] A. Klein, Unique continuation principle for spectral projections of Schrödinger operators and optimal Wegner estimates for non-ergodic random Schrödinger operators. Comm. Math. Phys. 323 (2013), 1229-1246. MR 3106507 Zbl 1281.47026

[25] A. Klein and A. Koines, A general framework for localization of classical waves: II. Random media. Math. Phys. Anal. Geom. 7 (2004), 151-185. MR 2057123 Zbl 1054.35102

[26] F. Klopp, Internal Lifshits tails for random perturbations of periodic Schrödinger operators. Duke Math. J. 98 (1999), 335-396. MR 1695202 Zbl 1060.82509

[27] F. Klopp, Weak disorder localization and Lifshitz tails. Comm. Math. Phys. 232 (2002), 125-155. MR 1942859 Zbl 1034.82024

[28] G. Lawler and Sokal, Bounds on the $\mathrm{L}^{2}$ spectrum for Markov chains and Markov processes: a generalization of Cheeger's inequality. Trans. Amer. Math. Soc. 309 (1988), 557-580. MR 0930082 Zbl 0716.60073

[29] G. A. Mezincescu, Lifschitz singularities for periodic operators plus random potentials. J. Statist. Phys. 49 (1987), 1181-1190. MR 0935490 Zbl 0971.82508

[30] C. Rojas-Molina, Etude mathématique des propriétés de transport des operatéurs de Schrödinger aléatoires avec structure quasi-cristalline. Ph.D. Thesis, Université de Cergy-Pontoise, Cergy-Pontoise, 2012. http://www.theses.fr/2012CERG0565

[31] C. Rojas-Molina, The Anderson model with missing sites. Oper. Matrices 8 (2014), 287-299. Zbl 06295133

[32] C. Rojas-Molina and I. Veselić, Scale-free unique continuation estimates and applications to random Schrödinger operators. Comm. Math. Phys. 320 (2013), 245-274. MR 3046996 Zbl 1276.47051

[33] C. Stolz, An introduction to the mathematics of Anderson localization. In R. Sims and D. Ueltschi (eds.), Entropy and the quantum II. Lecture notes from the $2^{\text {nd }}$ Arizona School of Analysis with Applications held at the University of Arizona, Tucson, AZ, March 15-19, 2010. Contemporary Mathematics 552. American Mathematical Society, Providence, R.I., 2011, 71-108. MR 2868042 MR 2868059 (collection) Zbl 1244.82031 Zbl 1223.82001 (collection)

[34] B. Simon and T. Wolff, Singular continuous spectrum under rank one perturbations and localization for random Hamiltonians. Comm. Pure Appl. Math. 39 (1986), 75-90. MR 0820340 Zbl 0609.47001

[35] W.-M. Wang, Localization and universality of Poisson statistics for the multidimensional Anderson model at weak disorder. Invent. Math. 146 (2001), 365-398. MR 1865399 Zbl 1035.82023

[36] F. Wegner, Bounds on the density of states in disordered systems. Z. Phys. B 44 (1981), 9-15. MR 0639135 
Received March 13, 2013

Alexander Elgart, Department of Mathematics, Virginia Tech, Blacksburg, VA, 24061, U.S.A.

E-mail: aelgart@vt.edu

A. Klein, University of California, Irvine; Department of Mathematics; Irvine, CA, 92697-3875, U.S.A.

E-mail: aklein@uci.edu 